\title{
NEPHROPROTECTIVE ACTIVITY OF ASPARAGUS RACEMOSUS AGAINST CISPLATIN-INDUCED NEPHROTOXICITY AND RENAL DYSFUNCTION IN EXPERIMENTAL RATS
}

\author{
YADAV AV*, UPASANI CD \\ Department of Pharmacology, SNJB's Shriman Sureshdada Jain College of Pharmacy, Chandwad, Maharashtra, India. \\ Email: yadavamruta4@gmail.com
}

Received: 26 June 2018, Revised and Accepted: 30 July 2018

\begin{abstract}
Objective: The current study was designed to evaluate the protective effect of standardized hydroalcoholic extract of Asparagus racemosus (AR) against cisplatin (CP)-induced nephrotoxicity in Wistar rats.

Methods: AR extract was administered orally at three dose levels (100, 200, and $400 \mathrm{mg} / \mathrm{kg}$ ). Vitamin E (250 mg/kg) was used as a standard nephroprotective agent. The kidney function test (estimation of serum creatinine, albumin, and blood urea nitrogen [BUN]), oxidative stress study (estimation of superoxide dismutase and malondialdehyde [MDA] activity), and histological examination of kidneys were conducted.

Results: The efficacy of AR was compared with CP-treated group. Serum creatinine and BUN were significantly (p<0.001) elevated in CP-treated group compared to control group. Hydroalcoholic extract of AR $(100,200$, and $400 \mathrm{mg} / \mathrm{kg}$ ) and Vitamin E (250 mg/kg) significantly (p<0.001) decreased the serum creatinine and BUN levels. CP exhibited significant $(\mathrm{p}<0.001)$ decrease in albumin when compared to control. Significant ( $<<0.001)$ increase in the serum albumin level was found in extract-treated group compared to CP group. Significant ( $<<0.001)$ decrease in activity of superoxide dismutase (SOD) was observed in the CP group as compared to control. AR (100 and $200 \mathrm{mg} / \mathrm{kg}$ ) significantly (p<0.01) increased SOD levels. AR (400 mg/kg) significantly $(\mathrm{p}<0.001)$ increased SOD levels. AR $(100,200$, and $400 \mathrm{mg} / \mathrm{kg})$ significantly $(\mathrm{p}<0.001)$ decreased MDA levels as compared to CP group. Histopathological examination of the kidneys showed that AR markedly ameliorated CP-induced renal tubular necrosis. Extract was found effective at all doses, although high dose (400 mg/kg) was found to be more effective and comparable with standard group (Vitamin E $250 \mathrm{mg} / \mathrm{kg}$ ).
\end{abstract}

Conclusion: The present investigation revealed that AR resulted in dose-dependent attenuation of CP-induced renal damage in rats.

Keywords: Asparagus racemosus, Cisplatin, Nephrotoxicity, Creatinine, Oxidative stress, Vitamin E.

(C) 2018 The Authors. Published by Innovare Academic Sciences Pvt Ltd. This is an open access article under the CC BY license (http://creativecommons. org/licenses/by/4. 0/) DOI: http://dx.doi.org/10.22159/ajpcr.2018.v11i12.28132

\section{INTRODUCTION}

Cisplatin (CP) is an effective antineoplastic DNA alkylating agent used for a wide variety of cancers. Even though the higher doses of CP are efficacious for the treatment of cancer, they are accompanied by many side effects including nephrotoxicity, neurotoxicity, bone marrow toxicity, gastrointestinal toxicity, and ototoxicity [1]. CP is a strong cellular toxin and nephrotoxicity is one of the most important complications of this drug in clinical and experimental models. The highest concentration of $\mathrm{CP}$ is observed in mitochondria, nuclei, cytosol, and microsomes. Nephrotoxicity induced by CP is mediated by mitogenactivated protein kinase (MAPK) intracellular signaling pathways. The MAPK pathways are activated by diverse extracellular physical and chemical stresses that regulate cell proliferation, differentiation, and survival [2]. Primary targets of $\mathrm{CP}$ in the kidney are the proximal and distal convoluted tubules where it accumulates and promotes cellular damage, by multiple mechanisms including oxidative stress, DNA damage, and apoptosis [1]. CP is interlaced to glutathione and metabolized through a gamma-glutamyl transpeptidase and cysteine S-conjugate $\beta$-lyase-dependent pathways to a reactive thiol The kidney accumulates CP by peritubular uptake and concentration of the drug in the renal cortex is several folds greater than other organs [2]. Platinum compounds mediate their cytotoxic effects by interaction with DNA. In an aqueous environment, the chloride ligands of $\mathrm{CP}$ are replaced by water molecules generating a positively charged electrophile. The electrophile reacts with nucleophilic sites on intracellular macromolecules to form DNA, RNA, and protein adducts. CP binds to DNA leading to the formation of inter- and intra-strand cross-links, thereby arresting DNA synthesis and replication in rapidly proliferating cells $[3,4]$. CP-induced oxidative stress and inflammatory response in the kidney may partially be prevented by several chemical and natural compounds such as antioxidant. Medicinal plants are a well source for variety of natural antioxidants and are used for the treatment of various diseases. Study of ancient classical Ayurvedic literature claimed several therapeutic attributes to the root of Asparagus racemosus (AR). Root of AR has been referred as bittersweet, emollient, constipating, galactagogue, aphrodisiac, diuretic, rejuvenating, carminative, stomachic, antiseptic, and as a tonic. Beneficial effects of the root of AR are suggested in nervous disorders, diarrhea, dysentery, tumors, hyperdipsia, neuropathy, cough, bronchitis, and certain infectious diseases [5,6]. The powdered dried root of AR is used in Ayurveda for dyspepsia [7]. It is also beneficial in the management of stress and inflammatory conditions $[8,9]$. The juice of fresh root of AR has been shown to have curative effect in patients with duodenal ulcers. It protects gastric mucosa against pentagastrin and carbachol-induced ulcers, by significantly reducing both severities of ulceration and ulcer index $[10,11]$. It is an important medicinal plant and its root paste or root juice has been used in various ailment and as health tonic $[12,13]$. Steroidal saponins (I-IV) are the active constituents found in Shatavari. Quantitative analysis revealed the presence of flavonoids (36.7 $\pm 3.9 \mathrm{mg} / 100 \mathrm{ml})$, polyphenols, $(88.2 \pm 9.3 \mathrm{mg} / 100 \mathrm{ml})$, and Vitamin C $(42.5 \pm 5.1 \mathrm{mg} / 100 \mathrm{ml})$. It is said to be a Rasayana herb in Ayurveda, i.e. group of plant drugs known to promote physical and mental health, improve defense mechanism of body [9].

AR has also proved to be effective in reducing hyperglycemia, creatinine, blood urea nitrogen (BUN), and oxidative stress in diabetic rats [14,15]. However, no scientific proof justifying the nephroprotective use of AR is available so far. Thus, the present investigation was undertaken 
to study the effect of the plant extract in CP-induced nephropathy in experimental rats.

\section{METHODS}

\section{Chemicals}

Standardized hydroalcoholic extract of AR (HAEAR) was procured from Shamantak enterprises, Pune. CP cis-diammineplatinum (II) dichloride was purchased from S.K. Enterprises, Pune. All other chemicals used were of the analytical grade.

\section{Animals}

A total of 36 Wistar male and female albino rats, weighing 200-250 g, were used for the study. The animals were maintained under standard laboratory conditions with controlled temperature $\left(20 \pm 2^{\circ} \mathrm{C}\right)$ and humidity (60\%) with regular light cycle (12 light/12 dark). The animals were acclimatized for 1 week before the study and had free access to standard laboratory food and water ad libitum. All experimental procedures were conducted in accordance with the principles for the care and use of laboratory animals in research and approved by the Institutional Animal Ethics Committee (ACP/ IAEC/2018/01).

\section{Experimental design}

The nephroprotective activity was tested on six groups of albino Wistar rats (3 males +3 females), each group consisting of six animals. Group I - Served as control received normal saline $(0.5 \%$; p.o) Group II - Toxic control rats received normal saline (0.5\%; p.o) and CP (6 mg/kg; i.p)

Group III - Received Vitamin E $250 \mathrm{mg} / \mathrm{kg}$ as standard nephroprotective agent and CP (6 mg/kg; i.p)

Group IV - Received HAEAR (100 mg/kg; p.o) and CP (6 mg/kg; i.p) Group V - Received HAEAR (200 mg/kg; p.o) and CP ( 6 mg/kg; i.p) Group VI - Received HAEAR (400 mg/kg; p.o) and CP (6 mg/kg; i.p)

On the $15^{\text {th }}$ day, $2 \mathrm{~h}$ after the administration of extracts and normal saline Groups II-VI received CP $(6 \mathrm{mg} / \mathrm{kg}$; i.p). At the end of the experimental period, i.e. on the $16^{\text {th }}$ day, rats were sacrificed by cervical dislocation. The blood was collected in an anticoagulant tube and allowed to stand for $30 \mathrm{~min}$ at $37^{\circ} \mathrm{C}$ and then centrifuged to separate the serum to evaluate the biochemical markers [16,17]. Serum malondialdehyde (MDA) was evaluated using the method of Buege. In this method, 100 $\mu \mathrm{L}$ serum was diluted to $500 \mu \mathrm{L}$ distilled water.

The samples were kept in boiling water bath for $15 \mathrm{~min}$. To the diluted sample, $1 \mathrm{ml}$ of trichloroacetic acid, thiobarbituric acid, and $\mathrm{HCl}$ reagent was added. The reaction mixture was cooled and centrifuged. The supernatant was taken and the optical density of the pink color formed was read at $535 \mathrm{~nm}$. The optical density of the pink color formed was directly proportional to the concentration of serum MDA in the given sample [18]. Serum superoxide dismutase activity was estimated by Marklund and Marklund method. In this method, superoxide anion is involved in auto-oxidation of pyrogallol at alkaline $\mathrm{pH}$ (8.5). The superoxide dismutase (SOD) inhibits auto-oxidation of pyrogallol. This can be determined as an increase in absorbance at $420 \mathrm{~nm}$ [19]. Jaffe'salkaline picrate method was used for creatinine estimation. Creatinine reacts with alkaline picrate to give an orange color. Intensity of color formed was directly proportional to the amount of creatinine present in the sample. Albumin determination was done by the Biuret method. Albumin present in globulin, free solution reacts with copper sulfate in alkaline medium to give violet color [20]. The BUN was calculated by Berthelot method. Urease catalyzes the conversion of urea to ammonia and carbon dioxide. The ammonia, thus released reacts with a mixture of salicylate, hypochlorite, and nitroprusside to yield indophenol, a blue-greencolored compound. The intensity of the color produced is directly proportional to the concentration of urea in the sample and was measured spectrophotometrically at $578 \mathrm{~nm}$ [21].

\section{Histopathology}

Fixation of the kidney was done by cutting and fixing in Bouin's fluid immediately after removal from the animal body. The tissues were fixed in Bouin's fluid for about $24 \mathrm{~h}$. The tissues were then taken and washed in glass distilled water for a day to remove excess of picric acid. This was followed by dehydration in which the tissues were kept in the following solutions for an hour each; 30\%, 50\%, 70\%, and 100\% alcohol. Xylene was used as the clearing agent, for 1 or $2 \mathrm{~h}, 2$ or 3 times. The tissues were removed out of xylene and were kept in molten paraffin embedding bath with molten wax maintained at about $50^{\circ} \mathrm{C}$. A clearglass plate was smeared with glycerine. L-shaped mold was placed on it to form a rectangular cavity. Molten paraffin wax was poured and air bubbles were removed using a hot needle. The tissue was placed in the paraffin and oriented on the surface to be sectioned. Then, the tissue was pressed gently toward the glass plate to make settle uniformly with a metal pressing rod and allowed the wax to settle and solidity room temperature. The paraffin block was kept in cold water for cooling. Section cutting was done with a rotatory microtome. The excess of paraffin around the tissue was removed by trimming, leaving $1 / 2 \mathrm{~cm}$ around the tissue. Then, the block was attached to the gently heated holder. To produce uniform sections, the microtome knife was adjusted to the proper angle in the knife holder with only the cutting edge coming in contact with the paraffin block. The tissue was cut in the thickness range of about $7 \mu \mathrm{m}$. The sections were spread on a warm water bath after they were detached from the knife with the help of hair brush. Required sections were spread on a clean slide and kept at room temperature. The sections were stained as follows: Deparaffinization with xylene 2 times each for $5 \mathrm{~min}$.

Dehydration is done through descending grades of ethyl alcohol. Staining with Ehrlich's hematoxylin was done for $15 \mathrm{~min}$. Then, sectioned tissues were thoroughly washed in tap water for 10 min rinsed with distilled water and stained with eosin. Dehydration is done again with ascending grades of alcohol. Finally, the tissues were cleared with xylene 2 times, each for about $3 \mathrm{~min}$ interval. On the stained slide, DPX mountant was applied uniformly and microglass cover slides were spread. Slides were observed in Nikon microscope and microphotographs were taken [22].

\section{Statistical analysis}

The results were expressed as mean \pm standard error of the mean. Comparison between groups was made by one-way analysis of variance followed by "Dunnett's test." $p<0.05$ was considered to be statistically significant.

\section{RESULTS}

\section{Biochemical analysis}

CP elicited a significantly increased creatinine levels and serum BUN levels. Serum creatinine was significantly $(p<0.001)$ elevated in the CP group when compared to control group. AR treatment $(100,200$, and $400 \mathrm{mg} / \mathrm{kg})$ and Vitamin E $(250 \mathrm{mg} / \mathrm{kg})$ significantly $(\mathrm{p}<0.001)$ decreased the serum creatinine levels as compared to the CP group. CP group exhibited significant $(p<0.001)$ decrease in albumin when compared to control. Significant $(p<0.001)$ increase in the serum albumin level was found when compared to the CP group. Serum BUN level was significantly $(\mathrm{p}<0.001)$ elevated in the CP group compared with control group. AR administration significantly $(\mathrm{p}<0.001)$ decreased the serum BUN level as compared with the CP control group. AR $(400 \mathrm{mg} / \mathrm{kg}$ ) was comparable to that of the standard group. Significant $(p<0.001)$ decrease in activity of SOD was observed in the CP group as compared to control. AR $(100$ and $200 \mathrm{mg} / \mathrm{kg})$ significantly $(\mathrm{p}<0.01)$ increased SOD level when compared to the CP group. AR (400 mg/ $\mathrm{kg})$ significantly $(\mathrm{p}<0.001)$ increased SOD levels. Significant $(\mathrm{p}<0.001)$ increase in activity of MDA was observed in the CP group as compared to control. AR $(100,200$, and $400 \mathrm{mg} / \mathrm{kg})$ significantly $(\mathrm{p}<0.001)$ decreased MDA levels as compared to the CP group. AR $(400 \mathrm{mg} / \mathrm{kg})$ exhibited results similar to that of standard Vitamin E (Table 1).

Histological examination of kidneys in normal control rats showed normal renal tubules and renal corpuscles. CP-treated group demonstrated histopathological damage with tubular degeneration, 
Table 1: Effect of HAEAR on biochemical parameters

\begin{tabular}{|c|c|c|c|c|c|}
\hline \multirow[t]{2}{*}{ Groups } & \multicolumn{5}{|l|}{ Parameters } \\
\hline & BUN (mg/dl) & Creatinine (mg/dl) & Albumin (g/dl) & MDA (nmol/mg) & SOD (U/mg) \\
\hline Control & $20.86 \pm 1.31$ & $0.70 \pm 0.04$ & $3.89 \pm 0.12$ & $7.61 \pm 0.19$ & $19.56 \pm 0.42$ \\
\hline $\mathrm{CP}+$ Vitamin E $250 \mathrm{mg} / \mathrm{kg}$ & $22.06 \pm 0.61^{b}$ & $0.83 \pm 0.05^{b}$ & $3.87 \pm 0.08^{\mathrm{b}}$ & $8.77 \pm 0.17^{b}$ & $18.98 \pm 0.26^{\mathrm{b}}$ \\
\hline $\mathrm{CP}+\mathrm{HAEAR} 100 \mathrm{mg} / \mathrm{kg}$ & $29.45 \pm 2.18^{\mathrm{b}}$ & $1.69 \pm 0.05^{b}$ & $4.03 \pm 0.07^{\mathrm{b}}$ & $7.65 \pm 0.09^{b}$ & $12.84 \pm 0.49^{c}$ \\
\hline $\mathrm{CP}+\mathrm{HAEAR} 200 \mathrm{mg} / \mathrm{kg}$ & $27.56 \pm 2.54^{b}$ & $1.49 \pm 0.97^{\mathrm{b}}$ & $3.02 \pm 0.05^{b}$ & $8.77 \pm 0.17^{b}$ & $11.89 \pm 0.12^{\mathrm{c}}$ \\
\hline CP+HAEAR $400 \mathrm{mg} / \mathrm{kg}$ & $22.68 \pm 0.54^{\mathrm{b}}$ & $0.98 \pm 0.18^{\mathrm{b}}$ & $3.13 \pm 0.21^{\mathrm{b}}$ & $8.33 \pm 0.21^{\mathrm{b}}$ & $15.57 \pm 0.15^{\mathrm{b}}$ \\
\hline
\end{tabular}

Values are expressed as mean \pm SEM ( $n=6$ ) (ANOVA followed by Dunnett's test). ${ }^{a} p<0.001$ when compared to control group, ${ }^{b} p<0.001$ when compared to CP group, ${ }^{c} \mathrm{p}<0.01$ when compared to CP group. SEM: Standard error of the mean, HAEAR: Hydroalcoholic extract of Asparagus racemosus, CP: Cisplatin, BUN: Blood urea nitrogen, MDA: Malondialdehyde, SOD: Superoxide dismutase, ANOVA: Analysis of variance

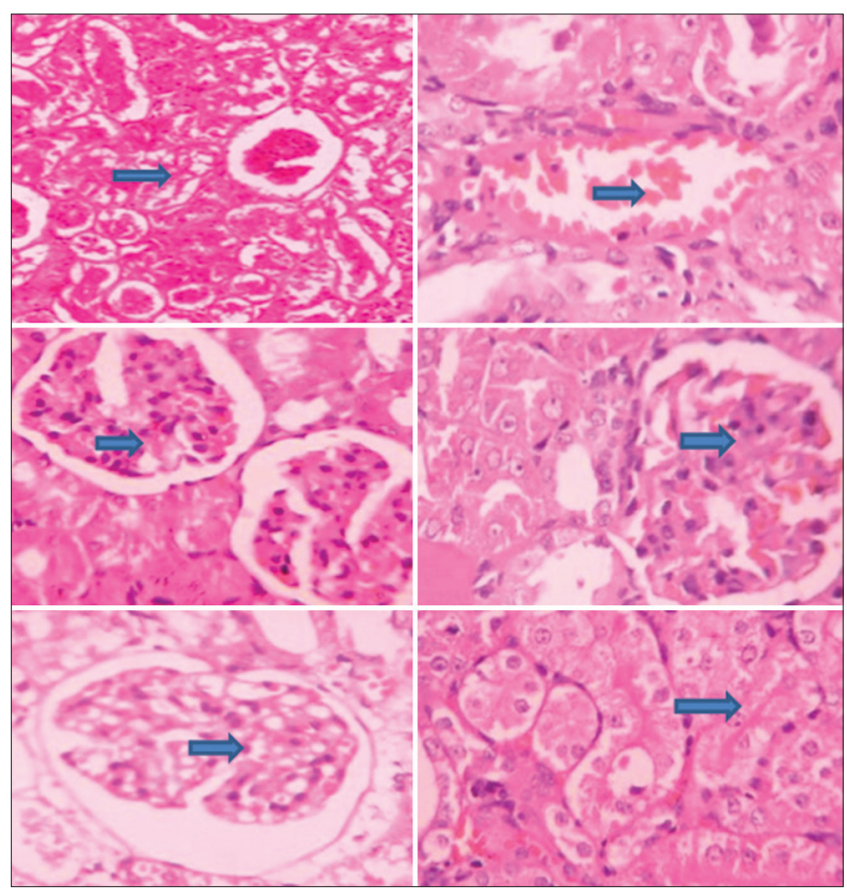

Fig. 1: Effect of hydroalcoholic extract of Asparagus racemosus on histopathology of kidneys. (a) Normal control; (b) cisplatin (CP); (c) CP+ Vitamin E $250 \mathrm{mg} / \mathrm{kg}$; (d) CP+ AR $100 \mathrm{mg} / \mathrm{kg}$; (e) CP+ AR $200 \mathrm{mg} / \mathrm{kg}$; (f) CP+AR $400 \mathrm{mg} / \mathrm{kg}$

tubular necrosis, and infiltration of inflammatory cells in the kidneys. $\mathrm{CP}$ intoxication showed severe atrophy of the glomerulus. Vitamin E-treated group showed marked improvement, showing normal kidney histology and architecture. AR (100 and $200 \mathrm{mg} / \mathrm{kg}$ ) showed marked improvement in comparison with the CP group. Abatement in the tubular necrosis and interstitial fibrosis was regarded. In AR ( $400 \mathrm{mg} / \mathrm{kg}$ ), diminution of tubular injury and atrophy was observed. It was evident by the normal histology of the kidney that was comparable to that of standard Vitamin E-treated group (Fig. 1).

\section{DISCUSSION}

The urinary tract is the body's drainage system for excretion of urine that is composed of wastes and extra fluid [23]. It also supports the elimination of nitrogenous waste produced by protein digestion from the bloodstream. The kidneys play an important role in maintaining normal blood $\mathrm{pH}$ by eliminating or maintaining acidic and basic compounds in the blood. Another important feature of the urinary system is the ability to distinguish between useful and toxic compounds in the blood that should be maintained or eliminated. The functional units called nephrons are capable of distinguishing between the different compounds dissolved in the blood, and eliminating only those that are not beneficial [24]. Numerous conditions such as diabetes, hypertension, glomerulonephritis, and autoimmune disease result in damage to the kidneys, thus affecting their ability to filter waste from the blood [25]. CP is used in the treatment of tumors of the neck, head, ovary, lung, and testicular cancer. The major limitation of CP is its ototoxic and nephrotoxic activity which limits its effectiveness [26]. CP exerts dose-dependent nephrotoxicity which limits its clinical usage in cancer chemotherapy.

Thrombotic microangiopathy, hypomagnesemia, salt wasting, Fanconilike syndrome, and anemia are other clinical manifestations of CP nephrotoxicity. It induces apoptosis and necrosis of renal tubular cells by activation of extrinsic and intrinsic mitochondrial pathways. It also involves p53-mediated proapoptotic activation of pro-inflammatory pathways. Pro-inflammatory pathways activation and infiltration of inflammatory cells are major mechanisms in CP-induced nephrotoxicity [27]. CP binds to DNA and forms inter- and intra-strand crosslinks, thus arresting DNA synthesis and replication [3]. The kidney accumulates CP to a greater extent unlike other organs and is the major route for its excretion. The $\mathrm{CP}$ concentration in proximal tubular epithelial cells is about 5 times the serum concentration [28]. The disproportionate accumulation of CP in kidney tissue contributes to CPinduced nephrotoxicity [29]. CP in kidneys penetrates tubular cells and concentrates mainly in the proximal tubules causing tubular damage. Tubular damage is characterized by a reduced glomerular filtration rate, increased serum creatinine and BUN, and decreased albumin levels [30].CP-induced nephropathy model also helps to understand the mechanisms involved in the induction of acute renal failure [35]. Toxins that cause tubular injury share many pathophysiological features with ischemic damage. Thus, CP potentially provides an excellent model not only for studying toxic nephrotoxicity but also ischemic nephrotoxicity [2]. CP (6 mg/kg) was sufficient to induce nephrotoxicity. Vitamin $\mathrm{E}$ was one of the antioxidant standard used to ameliorate CPinduced nephrotoxicity in rats. The present study reveals that the HAEAR possessed significant protective activity against CP-induced nephrotoxicity. Creatinine is produced from muscle metabolism. It is transported through the bloodstream to the kidneys. The kidneys filter out most of the creatinine and maintain the normal range of creatinine. Impairment in the function of kidneys results in the rise of creatinine level in the blood causing poor clearance of creatinine. Abnormally high levels of creatinine thus warn of possible malfunction of the kidneys. BUN is another indicator of kidney function. Urea is also a metabolic byproduct which is elevated if kidney function is impaired [32]. BUN level rises if kidney function decreases. Hypoalbuminemia is the strongest predictor of death in patients with renal failure. Albumin is the most abundant protein in nephrotic urine. Patients with lower serum albumin level have consistently higher morbidity rates [33]. Reduction in the GFR was indicated by increased levels of creatinine and BUN [34]. A significant decrease in serum albumin also indicated renal impairment. Administration of AR $(100,200$, and $400 \mathrm{mg} / \mathrm{kg})$ had beneficial effects on the kidneys treated with CP. This was evidenced by a significant decrease in the levels of creatinine and BUN in AR-treated group when compared to the CP group. It also augmented the reduced levels of albumin. Treatment with HAEAR markedly ameliorated the levels of creatinine and BUN indicating its renoprotective effect. SOD 
is the primary line of defense against free radical-induced oxidative stress. It is responsible for catalytic dismutation of highly reactive and potentially toxic superoxide radical to hydrogen peroxide [35]. Plants have evolved various protective mechanisms for minimizing deleterious effects of free radicals. The enzymatic defense comprises the efficient antioxidant enzymes such as catalase, peroxidase, and superoxide dismutase [36]

Increased ROS production in renal tissue may be responsible for damage of organs marked by changes in levels of MDA and SOD. MDA levels were significantly increased in rats treated with CP when compared to control group. AR significantly attenuated the MDA levels in renal tissue probably due to its capacity to scavenge oxygen free radicals in the kidneys. Moreover, it also significantly increased the levels of SOD resulting in improvement of kidney function and histopathology. Impairment in the histological features of the kidneys was substantiated by CP treatment. Our study demonstrated histological change in proximal and distal convoluted tubules, which were signs of tubular necrosis and atrophy of the vascular component in glomerulus in CP group. Vitamin E-treated rat kidney sections showed architecture similar to normal tubules. Recovery of renal function was observed by the treatment of AR evidenced by the regenerative capability of the renal tubules.

\section{CONCLUSION}

The study concludes that CP injury evidenced elevated biochemical markers and histopathological features of acute tubular necrosis. The administration of HAEAR resulted in dose-dependent attenuation of CPinduced renal damage. The nephroprotective potential may be due to its antioxidant properties. Further studies are required to characterize the phytoconstituents from AR and to study the exact mechanism of action.

\section{AUTHORS' CONTRIBUTION}

Both the authors contributed equally in preparing, editing, and reviewing the article.

\section{CONFLICTS OF INTEREST}

All authors declare that they have no conflicts of interest.

\section{REFERENCES}

1. Chatterjee P, Mukherjee A, Nandy S. Protective effects of the aqueous leaf extract of Aloe barbadensis on gentamicin and cisplatin induced nephrotoxic rats. Asian Pac J Trop Biomed 2012;2012:1754-63.

2. Hayati F, Hossainzadeh M, Shayanpour S, Abedi-Gheshlaghi Z, Beladi Mousavi SS. Prevention of cisplatin nephrotoxicity. J Nephropharmacol 2016;5:57-60.

3. Miller RP, Tadagavadi RK, Ramesh G, Reeves WB. Mechanisms of cisplatin nephrotoxicity. Toxins (Basel) 2010;2:2490-518.

4. Yao X, Panichpisal K, Kurtzman N, Nugent K. Cisplatin nephrotoxicity: A review. Am J Med Sci 2007;334:115-24.

5. Sharma PV, Charaka S. Chaukhambha Orientalis. India: Varanasi; 2001. p. 7-14.

6. Sairam K, Priyambada S, Aryya NC, Goel RK. Gastroduodenal ulcer protective activity of Asparagus racemosus: An experimental, biochemical and histological study. J Ethnopharmacol 2003;86:1-0.

7. Dalvi SS, Nadkarni PM, Gupta KC. Effect of Asparagus racemosus (Shatavari) on gastric emptying time in normal healthy volunteers. J Postgrad Med 1990;36:91-4.

8. Kanwar AS, Bhutani KK. Effects of Chlorophytum arundinaceum, Asparagus adscendens and Asparagus racemosus on pro-inflammatory cytokine and corticosterone levels produced by stress. Phytother Res 2010;24:1562-6.

9. Joshi T, Sah SP, Singh A. Antistress activity of ethanolic extract of Asparagus racemosus willd roots in mice. Indian J Exp Biol 2012;50:419-24

10. Shashi A, Jain SK, Verma A, Kumar M, Mahor A, Sabharwal M. Plant profile, phytochemistry and pharmacology of Asparagus racemosus (Shatavari): A review. Asian Pac J Trop Dis 2013;3:242-51.

11. Dahanukar SA, Date SG, Karandikar SM. Cytoprotective effect of
Terminalia chebula and Asparagus racemosus on gastric mucosa. Indian Drugs 1983;21:442-5.

12. Kirtikar KR, Basu BD. Indian materia medica. India 1975;3:2499-501.

13. Goyal RK, Singh J, Lal H. Asparagus racemosus-an update. Indian J Med Sci 2003;57:408-14.

14. Vadivelan R, Dipanjan M, Umasankar P, Dhanabal L, Palaniswami S, Muthureddy S, et al. Hypoglycemic, antioxidant and hypolipidemic activity of Asparagus racemosus on streptozotocin-induced diabetic in rats. Adv Appl Sci Res 2011;2:179-85.

15. Somania R, Singhai AK, Shivgunde P, Jain D. Asparagus racemosus willd (Liliaceae) ameliorates early diabetic nephropathy in STZ induced diabetic rats. Indian J Exp Biol 2012;50:469-75.

16. Yousef MI, Saad AA, El-Shennawy LK. Protective effect of grape seed proanthocyanidin extract against oxidative stress induced by cisplatin in rats. Food Chem Toxicol 2009;47:1176-83.

17. Debnath S, Babre N, Manjunath YS, Mallareddy V, Parameshwar P, Hariprasath K. Nephroprotective evaluation of ethanolic extract of the seeds of papaya and pumpkin fruit in cisplatin-induced Nephrotoxicity. J Pharm Sci Technol 2010;2:241-6.

18. Buege JA, Aust SD. Microsomal lipid peroxidation. Methods Enzymol 1978;52:302-10

19. Marklund S, Marklund G. Involvement of the superoxide anion radical in the autoxidation of pyrogallol and a convenient assay for superoxide dismutase. Eur J Biochem 1974;47:469-74

20. Rajagopal G, Toora BD. Practical Biochemistry. $2^{\text {nd }}$ ed. Lucknow: Ahuja Publishing House; 2010. p. 98-112.

21. Fawcett JK, Scott JE. A rapid and precise method for the determination of urea. J Clin Pathol 1960;13:156-9.

22. Bancroft D, Stevens A, Turner R. Theory and Practice of Histological Techniques. $4^{\text {th }}$ ed. Edinburgh, London, Melbourne: Churchill Livingstone; 1996. p. 53-83.

23. Health Information, The Urinary Tract \& How It Works. Available from: https://www.niddk.nih.gov/health-information/health-topics/ Anatomy/urinary-tract-how-it-works/Pages/anatomy.aspx. [Last cited on 2018 Feb 26]

24. Kirstin Hendrickson, Why is the Urinary System Important? Livestrong. com. Available from: http://www.livestrong.com/article/200414-whyis-the-urinary-system-important. [Last cited on 2018 Apr 28]

25. Kidney Disease, Labtestsonline. Org. Available from: https://www. labtestsonline.org/understanding/conditions/kidney/start/1. [Last cited on 2018 May 20].

26. Talukdar A, Kalita RD, GohainN, Saikia G, Kalita MC. Nephroprotective activity of the ethnomedicinal plants Caesalpinia bonduc and Momordica dioica from ne India against cisplatin induced chemotherapeutic toxicity. Int J Pharm Pharm Sci 2018;10:96-103.

27. Sahu BD, Kalvala AK, Koneru M, Mahesh Kumar J, Kuncha M, Rachamalla SS, et al. Ameliorative effect of fisetin on cisplatininduced nephrotoxicity in rats via modulation of NF- $\mathrm{\kappa B}$ activation and antioxidant defence. PLoS One 2014;9:1-15.

28. Kuhlmann MK, Burkhardt G, Köhler H. Insights into potential cellular mechanisms of cisplatin nephrotoxicity and their clinical application. Nephrol Dial Transplant 1997; 12:2478-80.

29. Arany I, Safirstein RL. Cisplatin nephrotoxicity. Semin Nephrol 2003;23:460-4

30. Ilic S, Stojiljkovic N, Veljkovic M, Veljkovic S, Stojanovic G. Protective effect of quercetin on cisplatin-induced nephrotoxicity in rats. Med Biol 2014;16:71-5

31. Balakumar P, Chakkarwar VA, Kumar V, Jain A, Reddy J, Singh M, et al. Experimental models for nephropathy. J Renin Angiotensin Aldosterone Syst 2008;9:189-95.

32. PhD Charles Patrick Davis. What are normal blood creatinine levels? Creatinine Blood Test: Normal Range, Low \& High Results. Medicine Net. Available from: http://www.medicinenet.com/creatinine_blood test/page2.htm. [Last cited on 2018 Feb 28]

33. Viswanathan V, Snehalatha C, Kumutha R, Jayaraman M, Ramachandran A. Serum albumin levels in different stages of Type 2 diabetic nephropathy patients. Indian J Nephrol 2004;14:89-92.

34. Domitrović R, Potočnjak I, Crnčević-Orlić Z, Škoda M. Nephroprotective activities of rosmarinic acid against cisplatin-induced kidney injury in mice. Food Chem Toxicol 2014;66:321-8.

35. Gautam RK, Singh RK, MS Karchuli. Evaluation of nephroprotective activity of Mentha Arvensis in cisplatin-induced nephrotoxicity. Asian J Pharm Clin Res 2014;7:188-91

36. Gupta SK, Najnin I, Choudhuri C, Mandal P. Elicitation of therapeutic potential and oxidative stress assessment of fenugreek sprouts under UV irradiation. Int J Pharm Pharm Sci 2017;9:91-9. 